\title{
Oficinas de intervenção psicossocial com agentes comunitários de saúde: reflexões e intervenções dialogadas
}

\author{
Psychosocial intervention workshops with community health workers: reflections and dialogued interventions

\section{Talleres de intervención psicosocial con agentes comunitarios de salud: reflexiones e intervenciones dialogadas}

Liliam das Graças Nascimento. Universidade Federal de São João del-Rei (UFSJ). São João-del Rei, MG, Brasil. saudepsic@yahoo.com.br (Autora correspondente)

Walter Melo. Universidade Federal de São João del-Rei (UFSJ). São João-del Rei, MG, Brasil. wmelojr@gmail.com

Marcos Vieira Silva. Universidade Federal de São João del-Rei (UFSJ). São João-del Rei, MG, Brasil.mvsilva@ufsj.edu.br

\section{Resumo}

Objetivo: trazer uma reflexão dos agentes comunitários de saúde (ACS) de um pequeno município da Região das Vertentes, no estado de Minas Gerais, sobre o próprio processo de trabalho. Métodos: pesquisa qualitativa, por meio de Oficinas de Intervenção Psicossocial (OIP) com 11 agentes comunitários de saúde ao longo de nove encontros. A análise de conteúdo, comparando a prática estabelecida e as diretrizes e determinações legais do Ministério da Saúde (MS) foi realizada a partir das seguintes categorias: a) Discrepância entre o que é determinado pelo MS e a prática dos ACS; b) Dilemas enfrentados pelos ACS; c) Qualidade do vínculo estabelecido entre ACS e usuários; e d) Vínculo dos ACS com os demais membros da equipe. Neste artigo são apresentados apenas os dados referentes à criação de vínculos com a comunidade e com a equipe, por se tratar de importante fator psicossocial para os trabalhadores da atenção primária. Resultados: a análise possibilitou o levantamento de algumas questões sobre 0 trabalho dos ACS: a sensação de desvalorização na equipe; a distância entre teoria e prática; a impossibilidade de se colocar em prática as determinações do MS; a necessidade de que a comunidade seja parceira do ACS; e a desinformação sobre o próprio trabalho. Conclusões: foi possível perceber que as principais modificações ocorridas no trabalho dos ACS dizem respeito a um maior detalhamento das atribuições determinadas pelo MS, à ênfase na contextualização de suas ações e ao aumento da burocratização que pode estar interferindo ou modificando sua prática.

\section{Abstract}

Objective: to promote a discussion among the community health workers (ACS) of a small city in the Region of Vertentes in the state of Minas Gerais, about their own work process. Methods: using qualitative research methods, we carried out Psychosocial Intervention Workshops (PIW) with 11community health workers during nine meetings. We conducted content analysis, comparing the established practice with the guidelines and legal regulations of the Brazilian Ministry of Health (MS), based on the following categories: a) discrepancy between what is determined by the MS and the practice of the ACS; b) dilemmas faced by the ACS; c) quality of the bond established between the ACS and the users; and d) relationships between the ACS and other team members. In this paper, we present only the data related to the creation of bonds with the community and with the team, because it is an important psychosocial factor for primary care workers. Results: based on the analyses, it was possible to raise some issues about the work of the ACS: the sense of devaluation within the team; the gap between theory and practice; the impossibility of putting into practice the determinations of the MS; the need for a partnership between the community and the ACS; and misinformation about the work itself. Conclusion: it was possible to realize that the major changes that occurred in the work of the ACS was related to a greater detailing of attributions determined by the MS, the emphasis in the context of their actions and increased bureaucratization that may be interfering with or modifying their practice.

\section{Palavras-chave:}

Sistema Único de Saúde

Agentes Comunitários de Saúde Saúde da Família

\section{Keywords:}

Unified Health System Community Health Workers Family Health 


\section{Resumen}

Objetivo: traer una reflexión de los Agentes Comunitarios de Salud (ACS) de un pequeño municipio de la Región de las Vertentes, en el estado de Minas Gerais, sobre el propio proceso de trabajo. Métodos: investigación cualitativa, por medio de Talleres de Intervención Psicosocial (TIP) con 11 agentes comunitarios de salud a lo largo de nueve encuentros. El análisis de contenido, comparando la práctica establecida y las directrices y determinaciones legales del Ministerio de la Salud (MS), fue realizada a partir de las siguientes categorías: a) Discrepancia entre lo que es determinado por el MS y la práctica de los ACS; b) Dilemas enfrentados por los ACS; c) Calidad del vínculo establecido entre ACS y usuarios; y d) Vínculo de los ACS con los demás miembros del equipo. En este artículo son presentados apenas los datos referentes a la creación de vínculos con la comunidad y con el equipo, por tratarse de importante factor psicosocial para los trabajadores de la atención primaria. Resultados: el análisis posibilitó el levantamiento de algunas cuestiones sobre el trabajo de los ACS: la sensación de desvalorización en el equipo; la distancia entre teoría y práctica; la necesidad de una colaboración entre la comunidad y los ACS; y la desinformación sobre el propio trabajo. Conclusión: fue posible percibir que las principales modificaciones ocurridas en el trabajo de los ACS dicen respeto a un mayor detalle de las atribuciones determinadas por lo MS, al énfasis en la contextualización de sus acciones y al aumento de la burocratización que puede estar interfiriendo o modificando su práctica.
Palabras clave:

Sistema Único de Salud Agentes Comunitarios de Salud Salud de la Familia

\section{Introdução}

A proposta de mudança no sistema público de saúde no Brasil foi marcada por experiências de práticas de açôes em saúde na própria comunidade. Essa proposta resultou no Programa de Agentes Comunitários de Saúde (PACS) e na Estratégia Saúde da Família (ESF). Estes são exemplos de importantes mudanças nos modelos assistenciais operados dentro do Sistema Único de Saúde (SUS). Esses modelos de prestação de serviços têm produzido modificaçóes na forma como são alocados os recursos, como são remuneradas as ações de saúde e no modo como são organizados os serviços. ${ }^{1}$

$\mathrm{Na}$ prática, o grande desafio é - e sempre foi - a superação de um modelo de assistência centrado na atenção médica para um modelo que entende a saúde de forma flexível e complexa, que requer, além da atenção curativa, ações de promoção da saúde e prevenção de doenças, considerando a complementaridade dessas açôes. O próprio conceito de saúde foi modificado e ampliado, passando a ser considerado para além da ausência de doenças. ${ }^{2}$

Conforme Viana e Dal Poz ${ }^{1}$ no início da década de 1990, o Ministério da Saúde institucionalizou as experiências de práticas em saúde com agentes comunitários que já estavam acontecendo, de forma isolada e focalizada, em várias regiōes do país. Em 1991, o PACS foi implantado em nível nacional e deu início ao enfoque na família, para além das práticas de saúde centradas no indivíduo. Além disso, esse programa apresenta uma visão da intervenção enquanto ação preventiva e instrumento de reorganização da demanda, primando pela participação da comunidade e integração desta com o serviço de saúde. O PACS foi formulado tendo como meta auxiliar na redução da mortalidade infantil e da mortalidade materna, em especial nas regiôes Norte e Nordeste, a partir da oferta dos serviços de saúde nas regiōes mais carentes e com menos recursos. A partir dos resultados obtidos pela ação do PACS, implantado no Ceará, em 1987, o Ministério da Saúde passou a considerar o ACS como um valioso integrante da organização do serviço de saúde local. ${ }^{1}$

O PACS não foi apenas mais um programa do Ministério da Saúde, mas um auxílio importante na implementação do SUS e na reorganização dos serviços locais de saúde. ${ }^{1,3}$ Ele mobilizou mudanças no sistema de saúde, pois exigiu que os municípios criassem condiçôes para a sua implantação: conselho de saúde ativo; Unidade Básica de Saúde (UBS) de referência do programa; profissional de saúde de nível superior para supervisionar e subsidiar as ações de saúde; e fundo municipal para receber os recursos destinados ao PACS. Foi necessária, então, a articulação entre os níveis do sistema (estadual e municipal) e os seus atores, o que constitui também uma importante estratégia para a reestruturação da atenção primária à saúde (APS).

O PACS facilitou o acompanhamento de grupos considerados vulneráveis (crianças, mulheres, idosos) e orientou o tipo de atendimento necessário e quais as principais demandas em cada contexto específico. Além disso, essa nova forma de atuação em saúde diminuiu a pressão das filas dos postos de saúde, possibilitando o atendimento dos casos mais simples na própria comunidade e prevenindo, por meio de orientaçóes educativas, doenças que levassem as pessoas a precisar de atendimento. ${ }^{4}$

Ao longo do tempo, os profissionais da ESF passaram a lutar, também, por melhores condiçôes de trabalho, revisão da carga horária, valorização profissional e reconhecimento. Os resultados das reivindicaçôes apontam para algumas modificaçôes que têm ocorrido, principalmente para o fato de o Agente Comunitário de Saúde (ACS) ser considerado o eixo de uma nova atenção primária à saúde. ${ }^{1}$ 
O ACS integra a equipe da ESF e se constitui como um elo fundamental entre a comunidade e o serviço de saúde. Em geral, ele é selecionado por meio de uma prova escrita sobre a ESF e suas atribuiçóes, sendo necessário que tenha acima de 18 anos e ensino médio completo. ${ }^{5} \mathrm{O}$ Conselho Estadual de Saúde de Minas Gerais destaca como principais atribuiçôes do ACS: desenvolver ações que objetivem a integração entre a equipe de saúde e a população adstrita à UBS; acompanhar as famílias cadastradas pertencentes à microárea; desenvolver ações educativas junto às famílias; orientar famílias quanto à utilização dos serviços de saúde disponíveis; e desenvolver atividades de promoção da saúde, prevenção das doenças e de agravos, e de vigilância à saúde, por meio de visitas domiciliares e de açóes educativas individuais e coletivas nos domicílios e na comunidade. ${ }^{5}$

O grande diferencial do ACS é incorporar a lógica e os valores da comunidade, por ser o membro da equipe de saúde que está mais próximo dela. Mas essa proximidade faz com que a população alimente expectativas em relação à ESF que são muito maiores do que esta pode atender. Para alguns autores ${ }^{3,6,7}$ é fundamental que a população esteja informada a respeito das atribuiçôes da ESF e sobre os recursos existentes no município, incluindo os serviços de emergência. Na maioria das vezes, exige-se muito do ACS e oferece-se pouco suporte à sua prática.

Estudos referentes aos ACS apontam para uma diversidade de assuntos que devem ser observados por esses trabalhadores, que ocupam o lugar de agenciadores entre os territórios dos saberes técnico e popular em saúde ${ }^{8}$ tendo que lidar com diversos saberes locais. ${ }^{9}$ Os ACS podem desempenhar funçôes em relação ao controle da tuberculose, ${ }^{10}$ ao uso de bebidas alcoólicas, ${ }^{11}$ ao cuidado com os idosos, ${ }^{12}$ à saúde fonoaudiológica da população, ${ }^{13}$ aos aspectos relacionados à saúde mental, ${ }^{14}$ à violência contra as mulheres ${ }^{15} \mathrm{e}$ ao uso indiscriminado de medicamentos. ${ }^{16}$ É evidenciada a importância das práticas educativas nas açôes dos ACS, ${ }^{17}$ enfatizando o trabalho de promoção da saúde bucal, ${ }^{18}$ assim como na formação do conceito de risco. ${ }^{19}$ As práticas dos ACS são referidas como elementos nucleares nas ações da ESF, mas que necessitariam de melhor instrumentalização, ${ }^{20}$ pois o cotidiano pode afetar-lhes, retirando-lhes a potência de agir. ${ }^{21}$ Levando esses aspectos em consideração, assim como as representaçôes dos ACS acerca de suas práticas assistenciais, ${ }^{22}$ que podem ganhar sentido ora no modelo biomédico, ora nos novos modelos de saúde, ${ }^{3}$ realizou-se uma Oficina de Intervenção Psicossocial (OIP) com o objetivo de possibilitar a reflexão sobre a prática dos ACS de um pequeno município na Região das Vertentes, no estado de Minas Gerais.

\section{Métodos}

A proposta da pesquisa foi apresentada, primeiramente, por meio de documento escrito ao secretário de saúde e à coordenadora da ESF do município. Após aceita, foi agendada uma reunião com os agentes comunitários para convidá-los a participarem da pesquisa. Todos os agentes se dispuseram a fazer parte, náo havendo resistências e nem desistências. As oficinas aconteceram de agosto a dezembro de 2009, em nove encontros quinzenais, às sextas-feiras, com duraçáo de uma hora e meia, com os onze agentes, lotados em duas equipes (uma rural e outra no centro da cidade). Os encontros foram realizados em ambiente neutro, cedido pela diretora da escola pública local, foram gravados,posteriormente transcritos e submetidos à análise de conteúdo. Foram debatidos os seguintes temas, de acordo com a Tabela 1.

Tabela 1. Temas debatidos com os agentes comunitários de saúde, Vertentes, MG, 2009.

Apresentação do objetivo da pesquisa

Quem é e o que faz o ACS

Questões éticas

Situações desafiantes da prática dos ACS

Leitura de documento do Ministério da Saúde sobre as 33 atribuições dos ACS

Dificuldades para seguir as determinações para o trabalho do ACS (em dois encontros)

Reflexão sobre o próprio trabalho (em dois encontros)

Fechamento das oficinas, com síntese dos debates.

A pesquisa utilizou metodologia qualitativa, considerada neste estudo como fundamental para a compreensão das dimensóes psicossociais do trabalho do ACS. Essa metodologia permitiu adentrar o campo de trabalho do ACS, buscando compreender o sentido desse trabalho para eles próprios, a forma como vivenciam essa prática, suas percepçóes e os valores atribuídos e utilizados para conduzir o dia-a-dia de trabalho. 
$\mathrm{Na}$ área da saúde, Minayo e Minayo ${ }^{23}$ consideram que os estudos devem utilizar metodologias qualitativas para apreenderem o significado e a intencionalidade peculiares às atitudes, relaçóes interpessoais e estruturas da sociedade. Há dados muito ricos a serem considerados ao se tentar entender os indivíduos e suas relaçóes. As dinâmicas de grupo em saúde são vistas como instrumento facilitador aos grupos que atuam nessa área e trabalham com a orientação dos usuários e com as práticas de prevenção e de promoção da saúde. ${ }^{24,25}$ Nesses possíveis processos, os trabalhadores podem adquirir um maior conhecimento sobre a construção do seu próprio ofício, as atividades dos demais profissionais e a qualidade do atendimento em saúde. As oficinas de dinâmica de grupo ${ }^{24,25}$ possibilitam o desenvolvimento de habilidades fundamentais ao atendimento em saúde, como a escuta, o enfrentamento de situaçôes de tensão e o aperfeiçoamento do diálogo. O grupo pode aprender a refletir sobre os objetivos de seu trabalho e sobre as próprias percepçóes em relaçáo à prática, bem como construir o conhecimento por meio da troca de experiências. O trabalho com o grupo possibilita a sensibilização, a reflexão e o desenvolvimento da criatividade dos integrantes de uma equipe.

Assim, optou-se pelo método da OIP porque esta articula a dimensão psicossocial (considera os atravessamentos políticos, sociais e institucionais), a dimensão clínica (trabalha com o projeto dos sujeitos) e a dimensão educativa (possibilita a informação, a apropriação de conteúdos, a reflexão, o processo de ressignificação, transmissão e conscientização), sem prescindir das técnicas grupais. Há nas OIP uma escuta diferenciada e uma apreensão dos significados atribuídos pelas pessoas, bem como dos afetos e das angústias despertados pela atuação grupal. ${ }^{24}$

A intervenção psicossocial tem como característica ser focal, voltando-se para problemas específicos, delimitados a partir do contato com o grupo. Esse tipo de intervenção segue a tradição da pesquisa-ação no campo da psicologia social. A relaçáo dos pesquisadores com as práticas de atenção e de intervenção é bastante forte, o que, associado ao conhecimento da efetividade da aplicação das OIP na saúde, também contribuiu para a escolha dessa metodologia. A intervenção psicossocial inclui a sensibilização em relação ao tema a ser trabalhado e a elaboração da experiência facilitada pela reflexão. Além disso, foi observada a importância da intervenção psicossocial com pequenos grupos nas áreas da saúde e comunidades em geral, como forma de facilitar que ocorram nos grupos as mudanças necessárias e desejáveis. ${ }^{25}$

Levando-se em consideração os temas de debate dos nove encontros e as atribuições conferidas aos ACS pelo Ministério da Saúde, foi realizada uma análise de conteúdo ${ }^{26}$ a partir das seguintes categorias: a) a discrepância entre o que é determinado e a prática do ACS; b) os dilemas enfrentados por esses profissionais; c) a qualidade do vínculo estabelecido entre os ACS e os usuários; e d) o vínculo dos ACS com os demais membros da equipe. Neste artigo são apresentados apenas os dados referentes à criação de vínculos com a comunidade e com a equipe, por se tratar de importante fator psicossocial, notadamente para os trabalhadores da atenção primária, como os ACS. A proposta é repensar o trabalho dos ACS levando-se em conta suas próprias concepçóes acerca do mesmo, as atribuiçóes determinadas pelo Ministério da Saúde e as produçóes teóricas acerca desse trabalho.

O Comitê de Ética da Universidade Federal de São João del-Rei (UFSJ) emitiu o parecer de no 008/2012 aprovando a presente pesquisa, e todos os ACS participantes assinaram o termo de consentimento livre e esclarecido (TCLE).

\section{Resultados e Discussão}

\section{0 agente comunitário de saúde como facilitador de vínculos}

É importante ressaltar a qualidade do vínculo do ACS com a comunidade. O texto "Do elo ao laço: o agente comunitário na construção da integralidade em saúde" 27 é muito ilustrativo quando se pensa na especificidade do trabalho do ACS. Como destacam os autores, os ACS trabalham "possibilitando a construção de um território comum, servindo como canais de comunicação, articulando escuta e fala - compreensão - entre pessoas da comunidade e profissionais da saúde” (p. 78) ${ }^{27}$. Nesta pesquisa foi possível corroborar essas ideias, pois os ACS manifestaram nas OIP a consciência da importância de seu papel enquanto facilitadores de vínculos, característica fundamental no trabalho na ESF junto à equipe. As ideias acerca do vínculo, surgidas durante as OIP, podem ser resumidas da seguinte maneira: a) o vínculo dos ACS com a comunidade é privilegiado pelo fato de estes pertencerem à população na qual atuam, conhecerem-na e terem acesso às residências; b) além disso, outra importante função do vínculo estabelecido pelos ACS é a facilitação do diálogo entre equipe de saúde e comunidade. 
Esse texto é muito importante, principalmente por apontar questôes sobre a percepção que o agente comunitário possui sobre os vínculos que constrói no dia-a-dia de sua atuação nas comunidades. Os autores ${ }^{27}$ lembram que o MS estabeleceu como premissa que, para se tornar agente comunitário, a pessoa deve residir na comunidade onde irá atuar, para facilitar a aproximação entre equipe de saúde e comunidade.

Segundo o Programa de Humanização do SUS, nas UBS e na prática dos ACS devem ser priorizadas as formas de acolhimento do usuário, sempre pautadas pelo exercício da solidariedade e da cidadania. Os ACS participantes da presente pesquisa reconheceram o diferencial do seu vínculo com a comunidade e com os outros profissionais da equipe, como destacado nas falas abaixo:

$O$ agente comunitário é um profissional importante na saúde porque ajuda os outros profissionais a conhecer melhor as familias atendidas e atender elas melhor também. (ACS 1).

O agente comunitário é quem leva informação e dá assistência à população sobre questóes relacionadas à saúde em geral. Faz com que o paciente se sinta uma pessoa importante porque tem alguém interessado em saber sobre a saúde dele. (ACS 4).

$O$ agente é o profissional que está sempre ajudando as pessoas, independente de serem pobres ou ricas. É quem faz os cadastros e orienta as pessoas sobre diabetes e pressão, por exemplo. (ACS 5).

$O$ agente comunitário é um amigo das familias... Ele chega e conhece aquela familia, sabe dos problemas que ela passa, procura ajudar a resolver o que tiver ao alcance. (ACS 5).

ACS significa muita coisa, é aquele que gosta de ajudar as pessoas, levando informaçóes e fazendo orientaçóes às pessoas da comunidade. Muitas vezes, tenta solucionar os problemas enfrentados pelas famílias. (ACS 7).

É interessante perceber a dimensão humanitária e de cidadania presente em documentos do Ministério da Saúde, que definem o ACS como sendo o "elo principal entre a comunidade e o serviço de saúde, o trabalhador de saúde que possui um perfil complexo, permeável, com fronteiras não bem definidas, fluidas, situado na interface entre as obrigaçôes do Estado para com seus cidadãos e o protagonismo da populaçáa". ${ }^{28}$

Durante os encontros, os ACS mencionaram os desafios de se lidar com pessoas, mas consideraram que o fato de conhecerem a comunidade é um facilitador da comunicação com as famílias:

Ah, a gente lida bem com as pessoas, elas têm liberdade de falar com a gente sobre os problemas do dia-a-dia. Porque também o agente é o amigo das familias: é aquele que participa da vida das pessoas, que deixa elas se abrirem e colocarem seus problemas, medos e preocupaçôes. (ACS 3).

Eu gosto de ser agente, mesmo que tem hora que as pessoas são muito ignorantes e nem sempre querem a nossa visita. Mas como a gente conhece a pessoa, você releva e tenta ajudar a pessoa assim mesmo. Ai outro dia que você volta a pessoa já atende melhor e a gente já sabe que ela tá num melhor humor. (ACS 9).

Silva et al. ${ }^{27}$ partindo da teoria de Habermas, comentam que o trabalho do agente comunitário estaria inserido em distintas formas do agir: a) o agir comunicativo, quando estabelece interaçóes no dia a dia da comunidade; e b) o agir estratégico, ao desenvolver, a partir de conhecimentos biomédicos, açôes de promoção de saúde ou prevenção às situaçôes de risco sanitário, tendo como meta atender às determinaçóes das políticas públicas de saúde. Os autores reconhecem que o êxito ou fracasso dependem dos meios oferecidos para o alcance dessa meta.

Na prática do ACS, é bastante comum o fato de a esfera do trabalho ser priorizada em detrimento da esfera das interaçóes. O excesso de burocratizaçáo, os protocolos e o tecnicismo são apontados como fatores que acabam enrijecendo o agir do ACS. Por outro lado, quando a esfera do trabalho consegue ser constantemente alimentada pela esfera das interaçóes, o trabalho do agente flui e passa a incluir diversas possibilidades em sua ação, tornando-se um laço nas relaçóes. ${ }^{27} \mathrm{~A}$ fala de um dos agentes comunitários é bem ilustrativa sobre a questão do tipo de ação que é enfatizado na prática:

Eu já trabalho como agente comunitário desde a primeira equipe que teve aqui, trabalho porque gosto mesmo de mexer com pessoas. Vejo que, quando a gente faz alguma coisa pra pessoa, ela fica feliz, agradece, manda Deus ajudar. E parece que eles gostam da gente. Mas mudou muito hoje em dia: o agente é muito cobrado, é muito papel para preencher. Tem de tudo quanto é tipo de formulário. E com isso a gente tem menos tempo para fazer as visitas, ou, dependendo a gente tem menos tempo para conversar nas famílias que a gente visita. Eu acho que mudou, mas quem saiu perdendo foi a população. Isso de fichas, a gente sabe que é só pra aumentar os números lá de cobertura. Mas e o atendimento? Como fica? (ACS 8). 
Outro agente, que também trabalha desde a constituiçâo da primeira equipe de saúde da família neste município, vê as mudanças - principalmente o excesso de burocracia - como um aspecto negativo da atual configuração do trabalho do ACS, mas acredita que algumas outras mudanças melhoraram a organização do serviço:

Eu achei bom quando fui selecionada para trabalhar nessa equipe. Ela é muito melhor do que a minha primeira equipe porque aqui parece que as pessoas levam mais a sério, ajudam mais uns aos outros. Têm mais compromisso mesmo. Eu acho que organizou mais o serviço. Antes era assim: quem queria trabalhava e quem não queria enrolava. O problema agora é muito formulário pra preencher e eu náo gosto muito dessa parte, mas os colegas me ajudam. Até nisso melhorou, viu? E lidar com esse povo é o que eu mais gosto de fazer. Só de ver que a pessoa ficou grata, a gente já se sente realizada. (ACS 7).

As OIP possibilitaram aos pesquisadores perceber que esses profissionais se sentem isolados dentro da equipe de saúde, ou seja, o vínculo estabelecido entre os próprios agentes ainda é frágil. No entanto, durante os encontros, os agentes demonstraram que necessitam de um espaço de reflexão e de debate que seja exclusivamente deles, para que, após conhecerem melhor o próprio papel, possam estabelecer novos vínculos com o restante da equipe e com os usuários. A partir do conhecimento da própria prática, os ACS poderão estabelecer laços, e não só se constituírem como elos entre a comunidade e o serviço de saúde local. Isso pode ser percebido na fala de um dos agentes:

A gente na equipe é o faz-tudo, mas é assim: o que dá certo é o que os outros da equipe fizeram e o que dá errado vem todo mundo cobrar da gente. O agente comunitário não é quase ninguém na equipe, mas a comunidade confia mais na gente do que nos outros, isso é verdade. Eu penso que se nos unirmos a situaçáo pode melhorar, porque a gente não tem confiança nem nos colegas (agentes de saúde), a gente vai ter nos outros (da equipe de saúde da família)? (ACS 8).

Os ACS participantes do grupo destacaram que há uma grande exigência por parte do Ministério da Saúde que repercute diretamente no relacionamento com a equipe. Não obstante, os agentes participantes da pesquisa apontam para a formaçáo de um grupo, no qual passaram a aceitar divergências de opiniōes. Assim, os ACS passaram a ouvir melhor os colegas de trabalho e a se sentirem mutuamente ajudados. O estabelecimento de um vínculo diferenciado será primordial para que os ACS realmente façam a diferença em sua prática.

A partir dos nove encontros promovidos nas Oficinas de Intervenção Psicossocial realizadas com os ACS, pôde-se verificar que se tratou de um modo eficaz de abordagem desse grupo de trabalhadores em saúde, principalmente por ser possível atingir o objetivo principal da presente pesquisa: conhecer a concepção dos ACS sobre o próprio trabalho. As OIP oportunizaram o encontro dos ACS e representaram o espaço aberto em que estes se sentiram à vontade para debater e refletir sobre os desafios e as conquistas de sua prática. Ao final dos encontros, os agentes perceberam que há muito mais aspectos comuns em sua prática do que imaginavam, embora haja diferenças econômicas, culturais e características peculiares de cada área de atuação. As oficinas não pretenderam torná-los um grupo com objetivos comuns definidos, mas possibilitar um espaço de encontro dos agentes, como mencionado anteriormente. A conclusão das oficinas foi de que os agentes passaram a perceber que as reivindicaçóes e os dilemas que guardavam, no dia-a-dia de trabalho, foram explicitados e compartilhados pelos demais integrantes do grupo.

O presente estudo tem como limitação principal o fato de não ser possível a generalização dos resultados para outros contextos, uma vez que objetivou o estudo de um contexto específico, através de estudo de caso. Por outro lado, pode-se dizer que a diretriz de descentralização do sistema de saúde foi realçada nesta pesquisa, dado que se trabalhou com agentes comunitários de saúde de um município com população de 4756 habitantes, com duas equipes de saúde, sendo uma urbana e a outra rural.

\section{Conclusão}

Ao se analisarem os documentos do Ministério da Saúde, ${ }^{29,30}$ pode-se perceber que as principais modificaçôes ocorridas no trabalho dos ACS dizem respeito a um maior detalhamento das atribuiçóes determinadas pelo MS, à ênfase na contextualização de suas açôes e ao aumento da burocratização de sua prática. O excesso de protocolos desafia o estabelecimento de vínculo, pois os ACS despendem parte de seu tempo preenchendo formulários, tempo que poderia ser investido no vínculo com a populaçáo, aspecto essencial do trabalho deste profissional.

O caminho traçado de pesquisa-ação possibilitou que os ACS adquirissem maior conhecimento sobre a atenção primária à saúde, especificamente através do trabalho do ACS e de suas açóes, que tão bem representam o atual momento da saúde pública no Brasil. 


\section{Referências}

1. Viana AL, Dal Poz MR. A reforma do Sistema Único de Saúde no Brasil e o Programa de Saúde da Família. PHYSIS: Rev Saúde Coletiva. 2005; 15 (supl.):225-64.

2. World Health Organization (WHO). Constitution of the World Health Organization. Basic Documents. Genebra: WHO; 1946.

3. Pupin VM, Cardoso CL. Agentes comunitários de saúde e os sentidos de "ser agente". Estudos de Psicologia. 2008;13(2):157-63. http://dx.doi.org/10.1590/S1413-294X2008000200008.

4. Senra TDM. Enfermagem na Saúde da Família. Apostila do Curso de Enfermagem da Universidade de Viçosa. Viçosa: [publisher unknown]; 2004.

5. Conselho Estadual de Saúde de Minas Gerais. Legislação do Sistema Único de Saúde - SUS. O SUS é para todos. Belo Horizonte: SESMG; 2006.

6. Chaves LM. Programa Saúde da Família: satisfação dos membros das famílias inscritas em Goiânia-GO [dissertação]. Rio de Janeiro: Universidade do Rio de Janeiro; 2002.

7. Silva MH; Santos MR. Perfil Social dos agentes comunitários de saúde vinculados ao programa de saúde da família da zona norte do município de Juiz de Fora. Rev APS. 2003;6(2):70-6.

8. Costa SL, Carvalho EN. Agentes comunitários de saúde: agenciadores de encontros entre territórios. Ciência Saúde Coletiva. 2012;17(11):2931-2940. http://dx.doi.org/10.1590/S1413-81232012001100010.

9. Lotta GS. Saberes Locais, Mediação e Cidadania: o caso dos agentes comunitários de saúde. Saúde Soc. 2012;(21):210-222.

10. Crispim JA, Scatolin BE, Silva LMC, Pinto IC, Palha PF, Arcêncio RA. Agente Comunitário de Saúde no controle da tuberculose na Atenção Primária à Saúde. Acta Paul Enferm. 2012;25(5):721-727.http://dx.doi.org/10.1590/S0103-21002012005000021.

11. Castanha AR, Araújo LF. Álcool e agentes comunitários de saúde: um estudo das representações sociais. Psico-USF. 2006;11(1):85-94.

12. Ferreira VM, Ruiz T. Atitudes e conhecimentos de agentes comunitários de saúde e suas relações com idosos. Rev Saúde Pública. 2012;46(5):843-849.http://dx.doi.org/10.1590/S0034-89102012000500011.

13. Santos JN. Percepção de agentes comunitários de saúde sobre os riscos à saúde fonoaudiológica. Rev Soc Bras Fonoaudiol. 2012;17(3):333-339. http://dx.doi.org/10.1590/S1516-80342012000300016.

14. Waidman MAP, Costa B,Paiano M. Percepções e atuação do agente comunitário de saúde em saúde mental. Rev Esc Enferm USP. 2012;46(5):1170-1177. http://dx.doi.org/10.1590/S0080-62342012000500019.

15. Hesler LZ, Costa MC, Resta DG, Colomé ICS. Violência contra as mulheres na perspectiva dos agentes comunitários de saúde. Rev Gaúcha Enferm. 2013;34(1):180-186. http://dx.doi.org/10.1590/S1983-14472013000100023.

16. Nunes CC, Amador TA, Heineck I. O medicamento na rotina de trabalho dos agentes comunitários de Saúde da Unidade Básica de Saúde Santa Cecília, em Porto Alegre, RS, Brasil. Saúde Soc. 2008;17(1):85-94.

17. Pereira IC, Oliveira MAC. O trabalho do agente comunitário na promoção da saúde: revisão integrativa da literatura. Rev Bras Enferm. 2013;66(3):412-419. http://dx.doi.org/10.1590/S0034-71672013000300017.

18. Bornstein VJ, David HMSL, Araújo JWG. Agentes comunitários de saúde: a reconstrução do conceito de risco no nível local. Interface Comunicação Saúde Educação. 2010;14(32):93-101. http://dx.doi.org/10.1590/S1414-32832010000100008.

19. Mialhe FL, Lefevre F, Lefreve AMC. O agente comunitário de saúde e suas práticas educativas em saúde bucal: uma avaliação qualiquantitativa. Ciênc Saúde Coletiva. 2011;16(11):4425-4432.

20. Costa SM, Araújo FF, Martins LV, Nobre LLR, Araújo FM, Rodrigues CAQ. Agente Comunitário de Saúde: elemento nuclear das ações em saúde. Ciênc Saúde Coletiva. 2013;18(7):2147-2156. http://dx.doi.org/10.1590/S1413-81232013000700030.

21. Galavote HS, Franco TB, Lima RCD, Belizário AM. Alegrias e tristezas no cotidiano de trabalho do agente comunitário de saúde: cenários de paixões e afetamentos. Interface Comunicação Saúde Educação. 2013;17(46):575-586. http://dx.doi.org/10.1590/S1414-32832013005000015

22. Baralhas M, Pereira MAO. Concepções dos agentes comunitários de saúde sobre suas práticas assistenciais. PHYSIS: Rev Saúde Coletiva. 2011;21(1):31-46.

23. Minayo MCS, Minayo CG. Difíceis e possíveis relações entre os métodos quantitativos e qualitativos nos estudos dos problemas de saúde. Rio de Janeiro: Fiocruz; 2001.

24. Afonso ML. Oficinas em Dinâmica de Grupo na Área da Saúde: um método de intervenção psicossocial. São Paulo: Casa do Psicólogo; 2006.

25. Oliveira EM,Spiri WC. Programa Saúde da Família: a experiência de equipe multiprofissional. Rev Saúde Pública. 2006;40(4):727-33. http://dx.doi.org/10.1590/S0034-89102006000500025.

26. Bardin L. Análise de conteúdo. Lisboa: Edições 70; 2006.

27. Silva RVB, Stelet BP, Pinheiro R, Guizardi FL. Do elo ao laço: o agente comunitário na construção da integralidade em saúde. In: Pinheiro R; Mattos RA, editors. Cuidado: as fronteiras da integralidade. Rio de Janeiro: Hucitec; 2004. p. 75-90.

28. Ministério da Saúde (BR), Secretaria Executiva. Programa Agentes Comunitários de Saúde (PACS). Brasília: Ministério da Saúde; 2001.

29. Ministério da Saúde (BR). Manual do Agente Comunitário de Saúde. Brasília: Ministério da Saúde, FUNASA; 1991.

30. Ministério da Saúde (BR). Programa de Agentes Comunitários de Saúde. Brasília: Ministério da Saúde, FUNASA; 1994. 